\title{
The accuracy of Johannesburg-based ambulance personnel in identifying stroke
}

\author{
D Nel,' BTEMC; W Stassen, ${ }^{1,2}$ MPhil EM \\ ' Department of Emergency Medical Care, Faculty of Health Sciences, University of Johannesburg, South Africa \\ ${ }^{2}$ Aeromedical Division, ER24 Emergency Medical Services, Johannesburg, South Africa
}

Corresponding author: W Stassen (stassen88@gmail.com)

Background. Stroke is a potentially life-threatening, time-dependent event that requires urgent management to reduce morbidity and mortality. It has been suggested that earlier recognition by ambulance personnel and transport to stroke centres may significantly reduce treatment delays. For this reason it is vitally important that ambulance personnel are able to accurately diagnose stroke.

Methods. A series of vignettes were created that included images, video and audio displaying either signs or symptoms of stroke or those of another condition. Ambulance personnel were asked to review each vignette and state whether the patient described was suffering from a stroke or not. Further investigation was sought by requesting each individual to motivate their answer, mentioning upon what their diagnosis was based.

Results. A total of 40 basic life support (BLS) and intermediate life support (ILS) personnel from different sites diagnosed 280 vignettes. BLS personnel were able to diagnose stroke with a sensitivity of $85.3 \%$ and a specificity of $89.9 \%$ (positive predictive value (PPV) $86.7 \%$, negative predictive value (NPV) $88.8 \%$ ), while ILS achieved a sensitivity of $98.2 \%$ and specificity of $94.0 \%$ (PPV 91.7\%, NPV 98.8\%). The combined sensitivity and specificity were $91.5 \%$ and $92.0 \%$, respectively (PPV 89.2\%, NPV 93.8\%). In order to aid their diagnosis, only $5 \%$ of BLS and $18.34 \%$ of ILS utilised validated stroke screening tools.

Conclusion. Despite not using validated screening tools, the ambulance personnel sampled in this study were able to identify stroke with high accuracy. Further studies should be considered to identify how these diagnoses were reached in order to identify training needs.

S Afr J Crit Care 2015;31(2):58-61. DOI:10.7196/SAJCC.2015.v31i2.247 

triple burden of disease, and an increase in lifestyle diseases such as stroke is becoming apparent. ${ }^{[1,2]}$ Within Africa, the prevalence of stroke has been estimated to be 81.2 (range 13.2 - 94.9)/100 000 person years ${ }^{[3]}$ Morbidity and mortality following stroke may be devastating, placing severe strain on healthcare infrastructure. Studies have shown that reduced time from insult to definitive treatment can greatly reduce the chances of dependency and death. ${ }^{[4]}$ It is understood that stroke must be identified early and the patient must be taken to the closest appropriate stroke facility for definitive treatment options. ${ }^{[4]}$ Often, prehospital personnel are the first healthcare professionals to come into contact with patients presenting with signs of stroke. ${ }^{[4,5]}$ From this, their role in the initial identification and diagnosis of stroke and appropriate referral to a stroke centre becomes clear. ${ }^{[4,6]}$ Early identification may significantly reduce the time to treatment, and finally translate to improved outcome. ${ }^{[7]}$ By allowing for preferential transport to stroke centres, patients may receive timely fibrinolysis or endovascular therapy to reduce cerebral tissue necrosis..$^{[4-6]}$

Locally, ambulance personnel are trained by means of a series of short courses. Formal stroke assessment is only taught within the intermediate life support (ILS) curriculum, unlike the international standard where emergency call-takers are expected to identify stroke immediately on first call to an emergency call centre. ${ }^{[4,6]}$ Prehospital personnel have been reported to identify stroke with an accuracy of between 61 and $83 \%$; however, in the presence of signs and symptoms that mimic stroke, a tendency towards overdiagnosis is seen.$^{[7]}$ In order to improve accuracy, two main prehospital stroke screening and diagnostic tools have been developed. By applying the Los Angeles Prehospital Stroke Scale (LAPSS) and the Cincinnati Prehospital Stroke Scale (CPSS), the accuracy may improve to sensitivities and specificities

of up to $74-97 \%$ and $72-94 \%$, respectively. ${ }^{[7-9]}$ Even though utilising these diagnostic tools is recommended by international guidelines, ${ }^{[4,6]}$ local data for the use and accuracy of prehospital stroke identification are not available.

In order to address this paucity in evidence available, the primary objective of this preliminary study was to determine the accuracy of basic life support (BLS) and ILS identification of stroke, and the rationale behind making these diagnoses. A secondary objective was to understand upon what these diagnoses were based.

\section{Methods}

A prospective, cross-sectional approach was employed by creating a series of seven non-validated vignettes that included images, video and audio displaying either signs or symptoms of stroke or those of other conditions. Each of the vignettes was created by including all elements of the LAPSS and CPSS into each case. This was confirmed independently during review by three emergency care practitioners. Table 1 shows the details of each vignette. Due to resource constraints, a non-probability convenience sample of 40 ambulance personnel was drawn from four different private emergency medical service stations within the Johannesburg region of Gauteng Province, South Africa (SA). The stations are located in the southern, eastern, northern and western parts of the greater Johannesburg area. Further investigation was done by requesting each individual to motivate their answer in an open-ended response, describing upon what their diagnosis was based.

Ambulance personnel were required to view a PowerPoint (Microsoft, USA) presentation of the vignettes (Table 1) and then mention whether stroke was present or absent and motivate how they reached the diagnosis. Three vignettes were positive for stroke diagnosis according to the LAPSS and CPSS, while four vignettes described patients with hypoglycaemia and dehydration, ptosis,

\section{Table 1. Vignettes}

\begin{tabular}{|c|c|c|c|}
\hline & Case description & Media used & $\begin{array}{l}\text { Stroke (based on } \\
\text { LAPSS/CPSS) }\end{array}$ \\
\hline Case 1 & $\begin{array}{l}\text { A } 70 \text {-year-old male patient with a history of hypertension is presented. The } \\
\text { patient has acute onset of depressed level of consciousness with anisocoria, } \\
\text { aphasia and unilateral facial droop. The patient also has hemiplegia. }\end{array}$ & $\begin{array}{l}\text { Anisocoria pictured. } \\
\text { Facial droop pictured. }\end{array}$ & Yes \\
\hline Case 2 & $\begin{array}{l}\text { A } 40 \text {-year-old female patient with dyspnoea is presented. She presents with } \\
\text { a productive cough and a physical examination in keeping with a lower } \\
\text { respiratory tract infection. Potentially mimicking facial droop, ptosis is seen. }\end{array}$ & Ptosis is pictured. & No \\
\hline Case 3 & $\begin{array}{l}\text { A 52-year-old female patient with a history of intense headache suddenly } \\
\text { presents with slurred speech and depressed level of consciousness. } \\
\text { Hemiparesis and facial droop is appreciable. Positive pronator drift is found. }\end{array}$ & $\begin{array}{l}\text { Facial droop is pictured. } \\
\text { Slurred speech is played. }\end{array}$ & Yes \\
\hline Case 4 & $\begin{array}{l}\text { A } 60 \text {-year-old male patient with depressed level of consciousness and general } \\
\text { weakness is presented. The patient has signs of acute gastroenteritis (with nausea, } \\
\text { vomiting and diarrhoea) and is a known diabetic. The patient is hypoglycaemic. } \\
\text { He has normal facial symmetry and appears acutely malnourished. }\end{array}$ & The patient is pictured. & No \\
\hline Case 5 & $\begin{array}{l}\text { A 28-year-old female patient with focal signs is presented. The patient is } \\
\text { known to suffer from depression and take selective serotonin-reuptake } \\
\text { inhibitors. She presents with tardive dyskinesia. All other findings are normal. }\end{array}$ & $\begin{array}{l}\text { Normal facies pictured. } \\
\text { Embedded video of tardive } \\
\text { dyskinesia shown. }\end{array}$ & No \\
\hline Case 6 & $\begin{array}{l}\text { An } 89 \text {-year-old frail patient with a history of atrial fibrillation is presented. The } \\
\text { patient has an acute history of facial asymmetry and aphasia. Hemiparesis is } \\
\text { also appreciable. }\end{array}$ & Assymetrical facies is shown. & Yes \\
\hline Case 7 & $\begin{array}{l}\text { A 32-year-old patient involved in a motor vehicle accident is presented. } \\
\text { The patient presents with ataxia, slurred speech and bilateral mydriasis. The } \\
\text { patient admits to alcohol use. }\end{array}$ & $\begin{array}{l}\text { Pupils are pictured. } \\
\text { An embedded video of ataxia } \\
\text { is shown. }\end{array}$ & No \\
\hline
\end{tabular}


tardive dyskinesia and ataxia secondary to alcohol intoxication. The data collection tool also collected demographic data on each participant.

Demographic data were analysed descriptively and reported in this manner (Table 2). The answers provided for each of the vignettes were marked according to the validated stroke scales (LAPSS and CPSS) by the principal investigators, and accuracy was determined by using standard formulae for sensitivity, specificity and negative and positive predictive values (NPVs and PPVs). Both the LAPSS and the CPSS were used as gold standard, i.e. a positive diagnosis for stroke in both scales was considered positive for stroke. The free text data were analysed to identify the diagnostic factors used to reach each diagnosis.

Each volunteer participant was required to provide written informed consent. Ethical approval was obtained from the University of Johannesburg's Human Research Ethics Committee (REC-01-1192014). All data collection forms were kept anonymous.

\section{Results}

A total of 43 questionnaires were distributed; three questionnaires were not completed as the participants were on duty and needed to service an emergency call. Only 40 questionnaires were therefore eligible for data analysis. A total sample of 280 vignettes was therefore analysed.

Tables 3 and 4 show the accuracies of stroke identification for BLS and ILS personnel, respectively. BLS were able to accurately diagnose stroke with a sensitivity of $85.3 \%$ and a specificity of $89.9 \%$ (PPV $86.7 \%$, NPV $88.8 \%$ ), while ILS achieved a sensitivity of $98.2 \%$ and a specificity of $94.0 \%$ (PPV 91.7\%, NPV 98.8\%). The combined sensitivity and specificity were $91.5 \%$ and $92.0 \%$, respectively (PPV 89.2\%, NPV 93.8\%).

Table 5 presents the factors on which stroke diagnoses were based. In general, there was a tendency by the sample at both levels of care (BLS and ILS) to rely on specific signs and symptoms, rather than using validated stroke screening tools such as CPSS and LAPSS. Only $5 \%$ of BLS and $18.3 \%$ of ILS utilised these tools to aid their diagnosis. In one instance, the application of the stroke scale was done incorrectly.

Participants incorrectly attributed presentations of patients with stroke to other causes such as patients being 'intoxicated' or 'drunk' on ethanol or patients having suffered a 'head injury.' Slurred speech was attributed to intoxication instead of stroke in certain instances; as was change in level of consciousness. Interestingly, a deranged level of consciousness was described as a sign that excludes stroke as a diagnosis. Left-sided hemiparesis was attributed to 'myocardial infarction' rather than stroke. This was more likely in the presence of abnormal heart rates and rhythms within the vignette.

\section{Discussion}

The study presented certain scenarios of various patients either having suffered from stroke (as identified by LAPSS and CPSS) or suffering from a condition that may mimic a stroke in presentation. BLS participants performed worse than the ILS participants. This was expected due to the fact that the ILS generally had more experience (mean 9 v. 3 years) and had more extensive medical training. In SA, both of these levels of care are educated by means of short-course certification. BLS personnel undergo a 6-week course that covers elementary aspects of prehospital care - this is the entry level for ambulance personnel. After 1000 practical patient hours as BLS, personnel are eligible for entry into the ILS course. The ILS course is
Table 2. Demographic information

\begin{tabular}{llll}
\hline Demographics & BLS & ILS & Total \\
\hline Male, $n$ & 18 & 8 & 26 \\
Female, $n$ & 2 & 12 & 14 \\
Average age (years) & 28 & 29 & 28.5 \\
Average work experience (years) & 3 & 9 & 6
\end{tabular}

Table 3. Stroke identification of BLS personnel

\begin{tabular}{|c|c|c|c|}
\hline & $\begin{array}{l}\text { LAPSS/CPSS: } \\
\text { Stroke, } n\end{array}$ & $\begin{array}{l}\text { LAPSS/CPSS: } \\
\text { No stroke, } n\end{array}$ & $\begin{array}{l}\text { Total } \\
n\end{array}$ \\
\hline BLS: Stroke & 52 & 8 & 60 \\
\hline BLS: No stroke & 9 & 71 & 80 \\
\hline Total & 61 & 79 & 140 \\
\hline
\end{tabular}

Table 4. Stroke identification of ILS personnel

\begin{tabular}{llll}
\hline & $\begin{array}{l}\text { LAPSS/CPSS: } \\
\text { Stroke, } \boldsymbol{n}\end{array}$ & $\begin{array}{l}\text { LAPSS/CPSS: } \\
\text { No stroke, } \boldsymbol{n}\end{array}$ & $\begin{array}{l}\text { Total, } \\
\boldsymbol{n}\end{array}$ \\
\hline ILS: Stroke & 55 & 5 & 60 \\
ILS: No stroke & 1 & 79 & 80 \\
Total & 56 & 84 & 140
\end{tabular}

Table 5. Frequency of reasons cited for stroke diagnosis

\begin{tabular}{lll}
\hline Diagnostic factor & BLS (\%) & ILS (\%) \\
\hline Hemiparesis & 61.7 & 80.0 \\
Abnormal pupils & 33.3 & 45.0 \\
Chronic hypertension & 60.0 & 45.0 \\
Confusion & 10.0 & 15.0 \\
Current hypertension & 28.3 & 25.0 \\
Decreased level of consciousness & 36.7 & 28.3 \\
Facial asymmetry & 41.7 & 68.3 \\
Hyperglycaemia & 12.5 & 12.5 \\
Patient age & 3.3 & 10.0 \\
Positive prediction by stroke scales & 5.0 & 18.3 \\
Slurred speech & 55.0 & 63.3 \\
Stroke scales & 5.0 & 18.3
\end{tabular}

over 18 weeks and teaches further skills such as intravenous therapy, basic electrocardiography and manual defibrillation.

Similar studies on stroke accuracy in SA could not be found. However, data from international studies may be comparable.

Studies have shown that EMS personnel can identify stroke with an accuracy of between 61 and $83 \% \cdot{ }^{[5,10,11]}$ A similar study conducted in Australia reported sensitivities of accurate paramedic stroke diagnosis of $93 \%$ and specificities of $87 \%$, which is similar to the accuracies identified by the current study. ${ }^{[12]}$ When using a validated tool (such as the LAPSS), the sensitivity and specificity may increase to $91 \%(95 \% \mathrm{Cl}$ $76-98 \%)$ and $97 \%(95 \% \mathrm{Cl} 93-99 \%)$, respectively. ${ }^{[13]}$

It is important to remember that many areas within SA remain underserviced by advanced life support, with $80 \%$ of all emergency care personnel being BLS. ${ }^{[14]}$ As first medical contact, these personnel 
need to be proficient at identifying stroke and expediting transport to stroke-ready hospitals. ${ }^{[4]}$ BLS may identify stroke accurately with a sensitivity of $85.3 \%$ and a specificity of $89.9 \%$ (PPV $86.7 \%$, NPV $88.8 \%$ ), but only use a validated stroke assessment tool $5 \%$ of the time. The literature suggests that diagnostic accuracy may increase should validated screening tools be used to aid diagnosis. Using these tools has been shown to consistently improve accuracy of stroke diagnosis. ${ }^{[7-9]}$ One of the manners in which the utilisation of a stroke diagnostic tool may be improved is by educational interventions. ${ }^{[5,15-17]}$ Yet, educating those in rural areas may be a costly exercise as they lack access to continuous professional development opportunities. ${ }^{[18]} \mathrm{An}$ online training programme has been suggested to counteract this, and has been shown to be of benefit. ${ }^{[19]}$ By allowing for earlier screening, transport may be expedited (including aeromedical transport), ${ }^{[4]}$ which might lead to shorter treatment times and a reduction in the burden of morbidity and mortality. ${ }^{[4,17]}$

Interestingly, the respondents (despite having high accuracy rates) incorrectly attributed clinical presentations to diagnoses. Positive clinical signs for stroke were often misattributed to non-stroke conditions or simply to age-related pathologies and found to be normal. This might show a critical need for development of the clinical reasoning abilities of local prehospital providers. The majority of the participants did not use any form of validated stroke screening tool to identify or rule out stroke. Shortcomings in the clinical decisionmaking of ambulance personnel have also been identified as one of the biggest threats to patient safety in the prehospital setting. ${ }^{[20]}$ Clinical reasoning and decision-making may be improved by way of simulation-based training. ${ }^{[21-23]}$

\section{Study limitations}

This preliminary study was largely observational and provides foundational data for future study. The role of sampling bias cannot be excluded. The sample was drawn from a single private service, and this detracts from the external validity. Internal validity is also compromised as the vignettes, even though they were developed and reviewed by senior emergency care practitioners, remained unvalidated.

\section{Conclusion}

It would be interesting to repeat this study using validated vignettes on a national, more heterogeneous sample. A project to determine the current knowledge of ambulance personnel on diagnostic stroke tools should be undertaken. A high accuracy of stroke identification was appreciable in this sample. This accuracy was higher than that of other studies cited, despite local personnel not utilising validated screening tools. It is concerning that clinical reasoning was inaccurate at times, and presentations were attributed to unrelated pathologies or comorbid conditions. More training should be considered to promote the use of these validated tools to aid in the diagnosis and referral of stroke patients.

\section{References}

1. Reed DM. The paradox of high risk of stroke in populations with low risk of coronary heart disease. Am J Epidemiol 1990;131(4):579-588.

2. Mbewu A. The burden of cardiovascular disease in sub-Saharan Africa. S Afr Heart 2009;6(1):4-10.

3. Adeloye D. An estimate of the incidence and prevalence of stroke in Africa: A systematic review and meta-analysis. PLoS One 2014;9(6):e100724. [http://dx.doi.org/10.1371/journal. pone.0100724]

4. Jauch EC, Saver JL, Adams HP, et al. Guidelines for the early management of patients with acute ischemic stroke. Stroke 2013;44:870-947. [http://dx.doi.org/10.1161/STR.0b013e318284056a]

5. Watkins CL, Leathley MJ, Jones SP, Ford GA, Quinn T, Sutton CJ. Training emergency services' dispatchers to recognise stroke: An interrupted time-series analysis. BMC Health Serv Res 2013;13:318. [http://dx.doi.org/10.1186/1472-6963-13-318]

6. Powers WJ, Derdeyn CP, Biller J, et al. 2015 AHA/ASA Focused Update of the 2013 Guidelines for the Early Management of Patients With Acute Ischemic Stroke Regarding Endovascular Treatment. Stroke 2015. [http://dx.doi.org/10.1161/STR.0000000000000074]

7. Bray JE, Martin J, Cooper G, Barger B, Bernard S, Bladin C. Paramedic identification of stroke: Community validation of the Melbourne ambulance stroke screen. Cerebrovasc Dis 2005;20(1):28-33. [http://dx.doi.org/10.1159/000086201]

8. Hurwitz AS, Brice JH, Overby BA, Evenson KR. Directed use of the Cincinnati Prehospital Stroke Scale by laypersons. Prehosp Emerg Care 2005;9(3):292-296. [http://dx.doi. org/10.1080/10903120590962283]

9. Chen S, Sun H, Lei Y, et al. Validation of the Los Angeles Pre-hospital Stroke Screen (LAPSS) in a Chinese urban emergency medical service population. PLoS One 2013;8(8):e70742. [http:// dx.doi.org/10.1371/journal.pone.0070742]

10. Smith WS, Isaacs M, Corry MD. Accuracy of paramedic identification of stroke and transient ischemic attack in the field. Prehosp Emerg Care 1998;2(3):170-175. [http://dx.doi. org/10.1080/10903129808958866]

11. Smith WS, Corry MD, Fazackerley J, Isaacs SM. Improved paramedic sensitivity in identifying stroke victims in the prehospital setting. Prehosp Emerg Care 1999;3(3):207-210. [http://dx.doi. org/10.1080/10903129908958938]

12. Bray JE, Coughlan K, Barger B, Bladin C. Paramedic diagnosis of stroke: Examining long-term use Bray JE, Coughlan K, Barger B, Bladin C. Paramedic diagnosis of stroke: Examining long-term use [http://dx.doi.org/10.1161/STROKEAHA.109.571836]

13. Kidwell CS, Starkman S, Eckstein M, Weems K, Saver JL. Identifying stroke in the field. Prospective validation of the Los Angeles Prehospital Stroke Screen (LAPSS). Stroke 2000;31(1):71-76. [http://dx.doi.org/10.1161/01.str.31.1.71]

14. Health Professions Council of South Africa. Professional Board for Emergency Care, iRegister. http://systems.hpcsa.co.za/iregister (accessed 7 Oct 2015).

15. Thomas $C$, Mackey $E$. Influence of a clinical simulation elective on Baccalaureate nursing student clinical confidence. J Nurs Educ 2012;51(4):236-239. [http://dx.doi.org/10.3928/01484834-20120224-03]

16. McNamara MJ, Oser $C$, Gohdes $D$, et al. Stroke knowledge among urban and frontier firs responders and emergency medical technicians in Montana. J Rural Health 2008;24(2):189-193. [http://dx.doi.org/10.1111/j.1748-0361.2008.00157.x]

17. Nam HS, Park E, Heo JH. Facilitating stroke management using modern information technology. J Stroke 2013;15(3):135-143.[http://dx.doi.org/10.5853/jos.2013.15.3.135]

18. Christopher L. An investigation into the non-compliance of Advanced Life Support practitioners with the guidelines and protocols of the professional board for emergency care practitioners. Research Report. Durban; Durban University of Technology, Department of Emergency Care and Rescue, 2007.

19. Stassen W, Wylie C, Holgate R. An online learning programme improves traumatic brain injury guideline adherence in a South African Helicopter Emergency Medical Service. Af J Emerg Med 2015; (in press).

20. Jensen J. Paramedic clincial decision-making: Results of two Canadian studies. Int Paramed Pract 2011;1(2):63-71. [http://dx.doi.org/10.12968/ippr.2011.1.2.63]

21. Aggarwal R, Mytton OT, Derbrew M, et al. Training and simulation for patient safety. Qual Saf Health Care 2010;19:i34-43. [http://dx.doi.org/10.1136/qshc.2009.038562]

22. Ten Eyck RP, Tews M, Ballester JM, Hamilton GC. Improved fourth-year medical student clinical decision-making performance as a resuscitation team leader after a simulation-based curriculum. Simul Healthc 2010;5(3):139-145. [http://dx.doi.org/10.1097/SIH.0b013e3181cca544]

23. Hagiwara MA, Kängström A. Effect of simulation on the clinical competence of Swedish ambulance nurses. Australas J Paramed 2014;11(2) 